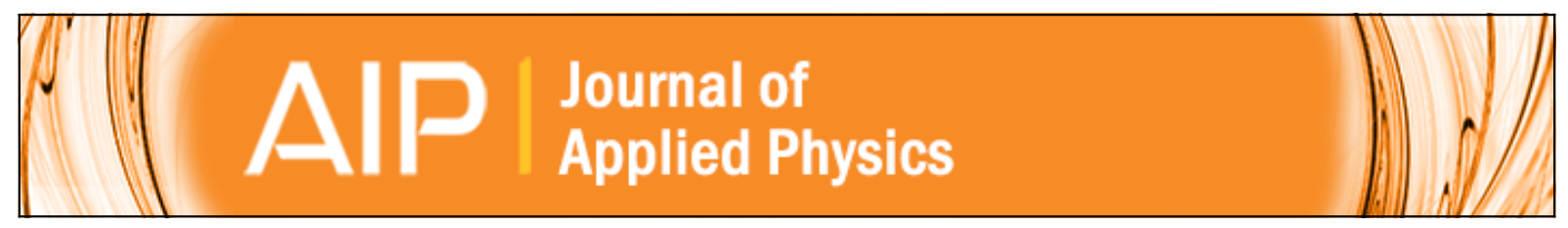

\title{
Photoelectric phenomena in polymer-based composites
}

A. Shik, H. Ruda, and E. H. Sargent

Citation: Journal of Applied Physics 88, 3448 (2000); doi: 10.1063/1.1289228

View online: $\mathrm{http}: / / \mathrm{dx}$.doi.org/10.1063/1.1289228

View Table of Contents: http://scitation.aip.org/content/aip/journal/jap/88/6?ver=pdfcov

Published by the AIP Publishing

\section{Articles you may be interested in}

Polymer-based solar cells having an active area of $1.6 \mathrm{~cm} 2$ fabricated via spray coating

APL Mater. 3, 126108 (2015); 10.1063/1.4937553

Self-assembly of single dielectric nanoparticle layers and integration in polymer-based solar cells

Appl. Phys. Lett. 101, 063105 (2012); 10.1063/1.4744928

Carrier transport in PbS nanocrystal conducting polymer composites

Appl. Phys. Lett. 87, 253109 (2005); 10.1063/1.2140885

Enhanced infrared photovoltaic efficiency in PbS nanocrystal/semiconducting polymer composites: 600-fold increase in maximum power output via control of the ligand barrier

Appl. Phys. Lett. 87, 233101 (2005); 10.1063/1.2137895

Microstructure-based numerical modeling method for effective permittivity of ceramic/polymer composites J. Appl. Phys. 97, 104104 (2005); 10.1063/1.1897071

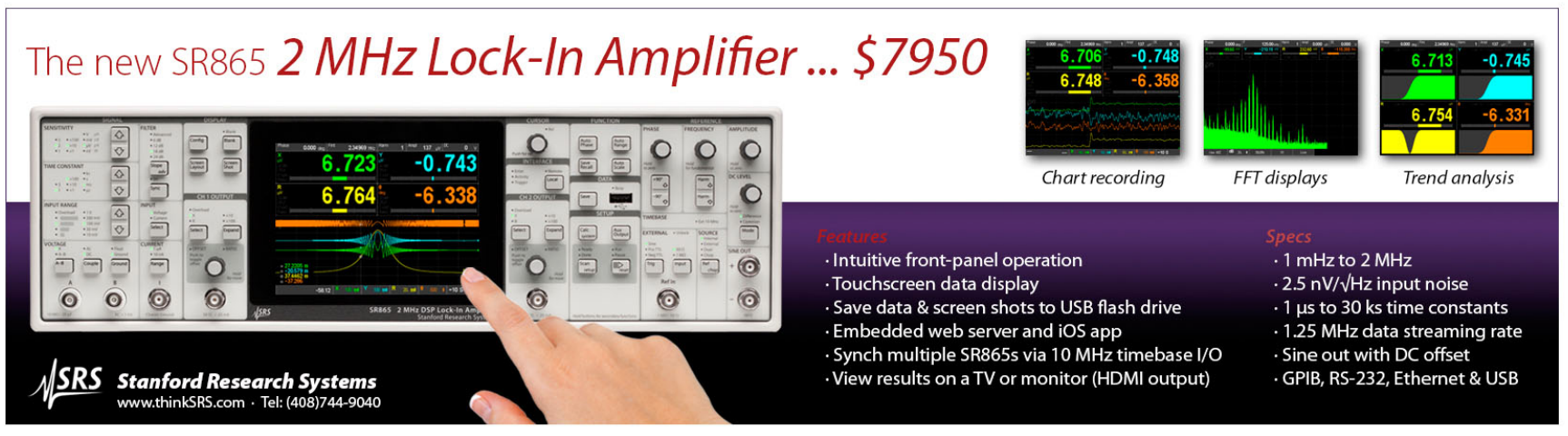




\title{
Photoelectric phenomena in polymer-based composites
}

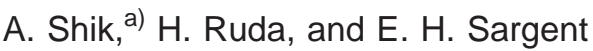 \\ University of Toronto, Toronto M5S 3E4, Canada
}

(Received 27 April 2000; accepted for publication 22 June 2000)

\begin{abstract}
We consider photoelectric phenomena in a composite medium in which inclusions form a type-II heterojunction with a host matrix. The model describes, in particular, polymer-based photosensitive structures with different types of semiconducting or insulating inclusions. It is shown that the separation on nonequilibrium carriers due to interface electric fields increases the hole component of photoconductivity, decreases the electron component, and results in a substantial increase of the total photoconductivity. The effect is observed experimentally in a wide range of polymer-based composites with disparate inclusions. For photodiode structures, this same phenomena either does not change, or slightly decrease, the quantum yield. (c) 2000 American Institute of Physics.
\end{abstract} [S0021-8979(00)03719-1]

\section{INTRODUCTION}

Conducting polymers provide a prospective basis for physically flexible, functionally versatile, low-cost, largearea electronic systems. Compared to inorganic elemental or compound crystals, the added degrees of freedom associated with molecular chemistry, structure, and organization broaden the range of functional application. At the same time, this added complexity poses new challenges in theory and fabrication.

Conducting polymers have benefited from extensive investigation over more than two decades. Polymer-based photoelectric devices (e.g., photoresistors and photodiodes) represent an important area for research. In spite of great progress made in this direction, the existing polymer-based devices still suffer from relatively low quantum yield. To a great extent, this may be attributed to the very low carrier mobility in polymers which prevents complete separation of photoexcited electron-hole pairs.

Recent experiments have shown that polymer photoconductivity can be improved by embedding semiconductor nanocrystals (including, but not limited to, CdS and CdSe), ${ }^{1,2}$ dielectric $\mathrm{TiO}_{2}$ nanocrystals, ${ }^{3}$ or fullerene $\mathrm{C}_{60}$ molecules ${ }^{4}$ within the polymer matrix. These "sensitizers" include disparate classes of materials-some conducting, others insulating; some absorbing/gain producting, others purely dispersive. It would seem that the photoconductivity enhancement has a general character associated with inclusion of embedded materials.

It has been proposed ${ }^{1-4}$ that an increase in photoconductivity may be related to charge separation at the interface between polymer matrix and the inclusions embedded therein. In this work, we consider the theory of this effect in detail, elucidate its general features, and determine its influence on the properties of composite-based photoconductors and photodiodes.

\footnotetext{
${ }^{a)}$ Electronic mail: shik@ecf.utoronto.ca
}

\section{MODEL BAND DIAGRAM}

An essential feature of conducting polymers used in photovoltaic devices is their relatively low electron affinity $\chi$, the energy separation between the vacuum level, and the lowest unoccupied molecular orbital (LUMO) (which plays the role of conduction band in conducting polymers). For the most popular conducting polymers-including polyphenylene vinylene (PPV), triphenyldiamine (TPD), polyvinyl carbazole $(\mathrm{PVK})$, oxadiazole derivative $(\mathrm{PBD})-\chi$ ranges from 2.3 to $2.6 \mathrm{eV}$. $^{5}$ This is less than typical electron affinities in $\mathrm{CdS}\left(3.8-4.8 \mathrm{eV}^{6}\right), \mathrm{CdSe}\left(4.0 \mathrm{eV}^{6}\right)$, and $\mathrm{TiO}_{2}(4.2$ $\mathrm{eV}^{3}$ ). Though the experimental values of $\chi$ even in wellstudied materials may vary from one source to another by several tenths of $\mathrm{eV}$ and for CdS even more, $\chi$ for polymers is nevertheless always much less than for all abovementioned materials. Investigations of the PPV-C 60 system ${ }^{4}$ also demonstrate that $\mathrm{C}_{60}$ is an electron acceptor for PPV.

In sum, in most polymer-based composites, the polymer component has a lower value of electron affinity than the imbedded material. The band gap of the latter can be larger than that of the polymer (as in $\mathrm{TiO}_{2}$ ) or smaller typically by only $0.1-0.5 \mathrm{eV}$ (as in CdS and CdSe). Given these parameters, the energy band diagram of the system has a form shown in Fig. 1(a). This is known in semiconductor structures as a type-II heterojunction. Thus, our aim will be to analyze the main light-induced regularities in composites consisting of the materials which form the type-II heterojunction. The model which we elaborate is applicable both to polymer-based mixtures and also to purely inorganic semiconductor quantum dot structures in type-II heterosystems, such as $\mathrm{In}_{1-x} \mathrm{Ga}_{x} \mathrm{As}_{\mathrm{S}} \mathrm{GaSb}_{1-y} \mathrm{As}_{y}{ }^{7}$ or $\mathrm{CdS}-\mathrm{PbS}{ }^{8}$ While a great number of publications are devoted to the type-I quantum dots with simultaneously confined electrons and holes, the properties of type-II dots, particularly, their photoelectric characteristics, remain almost uninvestigated.

In the presence of illumination the band diagram of Fig. 1(a) will be altered due to the charge of nonequilibrium carriers. During the initial period of excitation, electrons are captured by the potential well, giving rise to an accumulating 

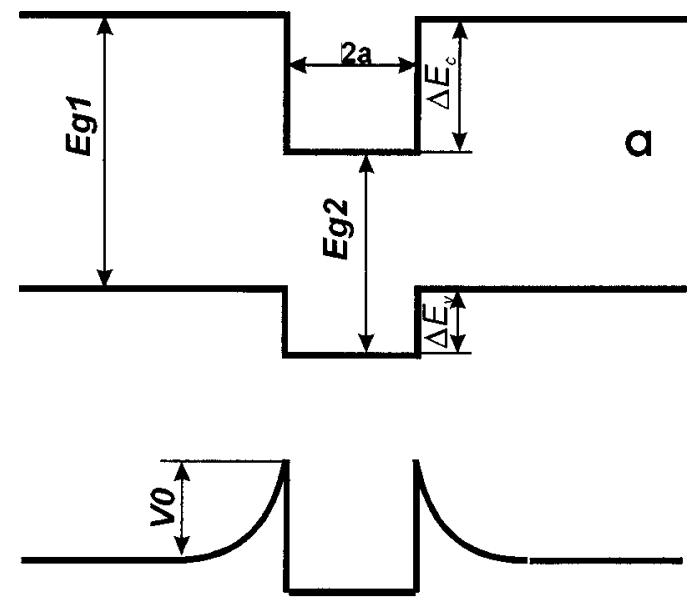

b

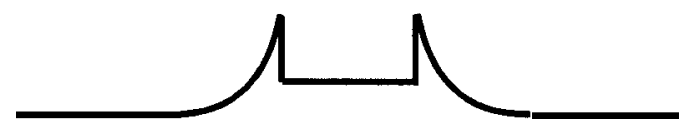

FIG. 1. Energy band diagram of a polymer-based composite (a) in equilibrium and (b) under illumination.

of negative charge $-Q$ therein. An equal number of holes in the surrounding matrix provide screening of this charge. The resulting band diagram is shown schematically in Fig. 1(b). If we assume the inclusion to have a spherical shape with the radius $a$, then the electric field at the interface

$$
\frac{d \varphi}{d r}(a)=\frac{Q}{\epsilon_{e} a^{2}},
$$

where $\epsilon_{e}$ is the dielectric constant of polymer matrix. In the close vicinity of the interface, the potential varies linearly. If the holes have a quasiequilibrium distribution, this results in an exponentially varying hole concentration: $p(r) \simeq p_{0} \exp$ $\left[-e Q(r-a) /\left(\epsilon_{e} a^{2} k T\right)\right]$. The number of holes concentrated in this region is given by

$$
P=\int p(r) \mathbf{d}^{3} \mathbf{r}=4 \pi \epsilon_{e} a^{4} k T p_{0} /(e Q) .
$$

The amplitude of band bending $V_{0}$ cannot be found from these approximate considerations, since this is determined by the distant potential tail rather than by the thin region with large $p$ close to the interface. $V_{0}$ is readily estimated from another consideration. Since the potential in accumulation layers varies logarithmically (see, e.g., Ref. 9), $V_{0}$ $\simeq k T \ln \left(l / r_{s}\right)$, where $l=N^{-1 / 3}$ is the average distance between inclusions. $N$ is the density of inclusions and $r_{s}$ $=\left(4 \pi p_{0} e^{2} / k T\right)^{-1 / 2}$ is the effective screening length.

In the absence of external current, the number of holes given by Eq. (2) must be equal to the number of electrons $Q / e$, which eventually gives the relationship between $Q$ and the hole concentration near the interface, $p_{0}$

$$
Q^{2}=4 \pi \epsilon_{e} a^{4} k T p_{0} \text {. }
$$

An independent equation, which links $Q$ with $p_{0}$ and allows them to be found separately, is deduced from recombination-generation carrier balance. The answer depends on the relationship between band gaps in the polymer matrix $E_{g}^{e}$ and in the inclusion $E_{g}^{i}$ and is derived below considering different possible cases. We assume that electrons in the conduction/LUMO band and holes in the valence/highest occupied molecular orbital (HOMO) band are in a quasiequilibrium and distributed in the potential relief according to the Boltzman formula. The validity of this assumption is discussed in Sec. III.

\section{A. Narrow-gap inclusions}

In polymer-based composites with semiconductor nanocrystals inclusions, such as PPV-CdS and PPV-CdSe, ${ }^{1,2,10,11}$ the valence band offset $\Delta_{v}$ is less than that in the conduction band $\Delta_{c}$. As a result, the recombination of electrons inside and holes outside inclusions occurs via hole activation over the potential barrier $\Delta_{v}$. If the light frequency $\omega$, with $E_{g}^{i}<\hbar \omega<E_{g}^{e}$, illuminates the structure, then both generation and recombination occur only inside inclusions. When the recombination rate described by the bimolecular quadratic expression $\gamma_{i} n p$, the balance between generation and recombination at steady state is given by

$$
\frac{\gamma_{i} p_{0} Q N_{v}^{i}}{N_{v}^{e}} \exp \left(-\frac{\Delta_{v}}{k T}\right)=\frac{4 \pi}{3} e a^{3} G_{i},
$$

where $G_{i}$ is the optical generation rate in the inclusions and $N_{v}^{i, e}$ are the effective densities of states in the valence bands of inclusion ( $i$ ) and matrix (e). Together with Eq. (3), this allows solution for the electron charge

$$
Q=\left(\frac{16 \pi^{2} e \epsilon_{e} a^{7} k T N_{v}^{e}}{3 \gamma_{i} N_{v}^{i}} G_{i}\right)^{1 / 3} \exp \left(\frac{\Delta_{v}}{3 k T}\right) .
$$

If $\hbar \omega>E_{g}^{e}$, the formula should be modified to reflect the fact that nonequilibrium carriers are generated mostly in the matrix rather than in the inclusions. This entails replacing $4 \pi a^{3} G_{i} / 3$ in Eqs. (4) and (5) by $G_{e} / N$.

\section{B. Wide-gap inclusions}

For dielectric wide-gap inclusions, such as, $\mathrm{TiO}_{2},{ }^{3}$ there are two main distinctions for the physical picture as compared with that of the previous subsection: (i) carriers are generated only in the polymer matrix; (ii) the energy barrier for electrons $\Delta_{c}$ is smaller than for holes $\Delta_{v}$, and recombination occurs predominantly in the matrix. If electrons and holes separately are in a quasiequilibrium, then the recombination rate at all points in the matrix has the same value $3 \gamma_{e} p_{0} Q N_{c}^{e} \exp \left(-\Delta_{c} / k T\right) /\left(4 \pi e a^{3} N_{c}^{i}\right)=G_{e}\left(N_{c}^{i, e}\right.$ are the corresponding densities of states in the conduction band). In combination with Eq. (3) this gives the expression similar to Eq. (5)

$$
Q=\left(\frac{16 \pi^{2} e \epsilon_{e} a^{7} k T N_{c}^{i}}{3 \gamma_{e} N_{c}^{e}} G_{e}\right)^{1 / 3} \exp \left(\frac{\Delta_{c}}{3 k T}\right) .
$$




\section{CHARGE SEPARATION AND PHOTOCONDUCTIVITY IN A POTENTIAL RELIEF}

We discuss the general features of charge separation in a semiconductor with a built-in nonuniform potential $V(\mathbf{r})$ having the characteristic spatial length $l$ and amplitude $V_{0}$.

The principal properties of nonequilibrium carriers in the system are determined by the relationship between the recombination time $\tau_{r}$ and the characteristic time of charge separation in internal electric fields $\tau_{s} \sim e l^{2} /\left(\mu V_{0}\right)$ where $\mu$ is the carrier mobility. If $\tau_{r}<\tau_{s}$, then drift in the internal electric field does not noticeably shift carriers during their lifetime, so that the carrier separation is negligible. In contradistinction, at $\tau_{r}>\tau_{s}$ the separation is essential and results in the formation of quasiequilibrium distributions of electrons and holes in the potential relief $V(\mathbf{r}) .{ }^{12}$ If the charge separation is negligible, $n=p=\sqrt{G / \gamma}$, so that $\tau_{r} \simeq 1 /(\gamma n)$ $\simeq 1 / \sqrt{\gamma G}$ and the condition $\tau_{r}>\tau_{s}$ can be written as

$$
\frac{e l^{2} \sqrt{\gamma G}}{\mu V_{0}}<1 .
$$

Even for very low carrier mobilities typical of conducting polymers, Eq. (7) is satisfied at sufficiently low enough light intensities. The carriers then obey a quuasiequlibrium distribution.

If a strong inequality opposite to Eq. (7) is satisfied, then the quantum yield of photosensitive devices, especially photodiodes considered in Sec. IV, is very low. This case is of little practical interest, and we therefore restrict our analysis to the quasiequilibrium case Eq. (7). The use of the Boltzmann distribution in describing occupation statics also depends on these conditions being satisfied.

In the absence of current, and with carriers in quasiequilibrium, electrons and holes can be described by the constant quasi-Fermi levels $\zeta_{n}$ and $\zeta_{p}$ which can be directly obtained from Eqs. (5) or (6). Measuring energies from the inclusion conduction band edge (see Fig. 1), the quasi-Fermi levels in the cases of narrow-gap inclusions illuminated under the condition $E_{g}^{i}<\hbar \omega<E_{g}^{e}$

$$
\begin{aligned}
& \zeta_{n}=\frac{\Delta_{v}}{3}+\frac{1}{3} \ln \frac{9 k T \epsilon_{e} N_{v}^{e} G_{i}}{4 \pi e^{2} a^{2} \gamma_{i} N_{v}^{i}\left(N_{c}^{i}\right)^{3}} ; \\
& \zeta_{p}=-E_{g}^{e}+\Delta_{c}-\frac{2 \Delta_{v}}{3}-\frac{1}{3} \ln \frac{4 \pi e^{2} a^{2}\left(N_{v}^{e}\right)^{2} G_{i}^{2}}{9 \gamma_{i}^{2} \epsilon_{e}\left(N_{v}^{i}\right)^{2}\left(N_{v}^{e}\right)^{3}} .
\end{aligned}
$$

As previously mentioned, when $\hbar \omega>E_{g}^{e}, 4 \pi a^{3} G_{i} / 3$ is replaced by $G_{e} / N$. For wide-gap inclusions

$$
\begin{aligned}
& \zeta_{n}=\frac{\Delta_{c}}{3}+\frac{1}{3} \ln \frac{9 k T \epsilon_{e} G_{e}}{4 \pi e^{2} a^{2} \gamma_{e} N_{c}^{e}\left(N_{c}^{i}\right)^{2}} ; \\
& \zeta_{p}=-E_{g}^{e}+\frac{\Delta_{c}}{3}-\frac{1}{3} \ln \frac{4 \pi e^{2} a^{2}\left(N_{c}^{i}\right)^{2} G_{e}^{2}}{9 \gamma_{e}^{2} \epsilon_{e}\left(N_{c}^{e}\right)^{2}\left(N_{v}^{e}\right)^{3}} .
\end{aligned}
$$

To calculate the photoconductivity, we must take into account the detailed spatial distribution of electron and hole currents. The inclusions are characterized by a very high

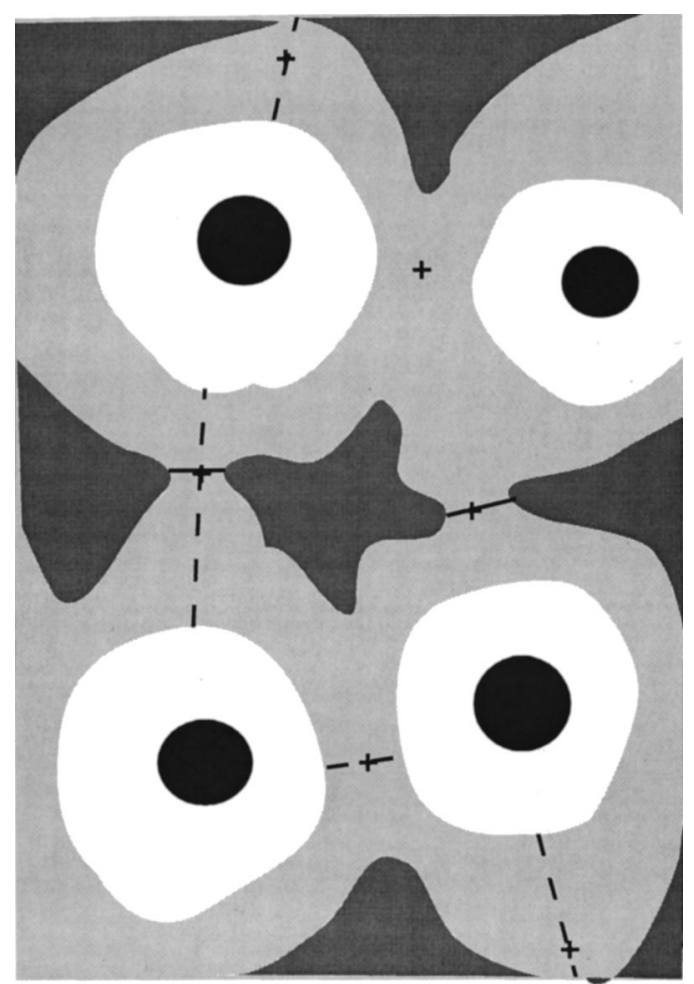

FIG. 2. Potential and concentration distribution in a polymer-nanocrystal system. Darker areas represent regions of higher electron and lower hole concentration. Black circles depict inclusions. Percolation pathways for electrons and holes are shown by solid and broken lines, respectively. The saddle points which dominate electron and hole conductivities are marked by crosses.

electron concentration, in principle favoring conduction, but their nearest neighborhood presents a high barriers for electrons. For this reason, the electron current is determined by the regions far from inclusions. Here, the electrical potential and electron concentration are maximal relative to the rest of the matrix.

In contrast, the hole current is carried by the spherical shells surrounding the inclusions, and through the local potential minina connecting them (Fig. 2). For both electrons and holes, the saddle region of maximal resistance which determines the current lies approximately halfway between neighboring inclusions. The electron and hole energies in this region, which in fact represent percolation levels $\epsilon_{n, p}$ in the conduction and valence bands (see, e.g., Ref. 13), are approximately equal to $\Delta_{c}-V_{0}$ and $\Delta_{c}-E_{g}^{e}-V_{0}$, respectively. (To be more exact, for a random position of inclusions, the saddle points for electrons and holes do not coincide and have slightly higher carrier concentrations which is typical for a three-dimensional percolation. ${ }^{14}$ However, for a logarithmically varying potential $V(\mathbf{r})$ this factor is of a minor importance and for our semiquantitative analysis can be neglected.)

To obtain the electron and hole photoconductivities of the composite system, we note that $V_{0} \simeq k T \ln \left(l / r_{s}\right)$, and $p_{0}$ which determines $r_{s}$ is given by Eqs. (3), (5), and (6). We find 


$$
\begin{aligned}
\sigma_{n} & \simeq e \mu_{n} N_{c}^{e} \exp \left(\frac{\zeta_{n}-\epsilon_{n}}{k T}\right) \\
& \simeq \frac{e \mu_{n} N_{c}^{e}}{N_{c}^{i}}\left(\frac{12 \pi \sqrt{\epsilon_{e}} e^{2}\left(N_{v}^{e}\right)^{2}}{a k T \gamma_{i}^{2}\left(N_{v}^{i}\right)^{2} N} G_{i}^{2}\right)^{1 / 3} \exp \left(\frac{2 \Delta_{v}-3 \Delta_{c}}{3 k T}\right) \\
\sigma_{p} & \simeq e \mu_{p} N_{v}^{e} \exp \left(\frac{\epsilon_{p}-\zeta_{p}}{k T}\right) \\
& \simeq \mu_{p}\left(\frac{a e k T N_{v}^{e} N}{12 \pi \sqrt{\epsilon_{e}} \gamma_{i} N_{v}^{i}} G_{i}\right)^{1 / 3} \exp \left(\frac{\Delta_{v}}{3 k T}\right)
\end{aligned}
$$

for $E_{g}^{i}<\hbar \omega<E_{g}^{e}$;

$$
\begin{aligned}
\sigma_{n} \simeq & \frac{3 e \mu_{n} N_{c}^{e}}{N_{c}^{i} a^{2} N}\left(\frac{\sqrt{\epsilon_{e}} e^{2}\left(N_{v}^{e}\right)^{2}}{4 \pi a k T \gamma_{i}^{2}\left(N_{v}^{i}\right)^{2}} G_{e}^{2}\right)^{1 / 3} \\
& \times \exp \left(\frac{2 \Delta_{v}-3 \Delta_{c}}{3 k T}\right), \\
\sigma_{p} \simeq & \mu_{p}\left(\frac{e k T N_{v}^{e}}{16 \pi^{2} \sqrt{\epsilon_{e}} a^{2} \gamma_{i} N_{v}^{i}} G_{e}\right)^{1 / 3} \exp \left(\frac{\Delta_{v}}{3 k T}\right)
\end{aligned}
$$

for $E_{g}^{i}<E_{g}^{e}<\hbar \omega$ and

$$
\begin{aligned}
\sigma_{n} & \simeq \frac{e \mu_{n} N_{c}^{e}}{N_{c}^{i}}\left(\frac{12 \pi \sqrt{\epsilon_{e}} e^{2}\left(N_{c}^{i}\right)^{2}}{a k T \gamma_{e}^{2}\left(N_{c}^{e}\right)^{2} N} G_{e}^{2}\right) \exp \left(\frac{2 \Delta_{v}-3 \Delta_{c}}{3 k T}\right), \\
\sigma_{p} & \simeq \mu_{p}\left(\frac{a e k T N_{c}^{i} N}{12 \pi \sqrt{\epsilon_{e}} \gamma_{e} N_{c}^{e}} G_{e}\right)^{1 / 3} \exp \left(\frac{\Delta_{c}}{3 k T}\right)
\end{aligned}
$$

for $E_{g}^{e}<\hbar \omega<E_{g}^{i}$.

It is worthwhile to compare these expressions with that for the photoconductivity of uniform matrix in the absence of potential relief: $\sigma_{n, p}^{0}=e \mu_{n, p} \sqrt{G_{e} / \gamma_{e}}$. If the band offsets $\Delta_{n, p} \gg k T$, then, due to exponential factors in Eqs. (10)-(15), $\sigma_{n} \ll \sigma_{n}^{0}$ but $\sigma_{p} \gg \sigma_{p}^{0}$. Thus, except for the case of anomalously low $\mu_{p}$ compared to $\mu_{n}$, the presence of inclusions increases photoconductivity of the system, which, in this case, occurs purely by hole conduction.

Similar photoconductivity growth occurs in chemically uniform semiconductors with a random electrostatic potential $V(\mathbf{r})$ caused by strong compensation, irradiation, grain boundaries, etc. ${ }^{12}$ The effect is caused by charge separation in the built-in potential $V(\mathbf{r})$, which created a potential barrier for recombination $\Delta_{r}$ exponentially increasing the lifetime and, hence, the concentration of nonequilibrium carriers. Though $V(\mathbf{r})$ also creates also a potential barrier for carrier transport, thereby suppressing conductivity, this drift barrier $\Delta_{d}$ is less that $\Delta_{r}$ so that their joint influence results in increased photoconductivity. In the case of the two-phase system considered herein, similar arguments can be applied. For holes, the connection comes via $\Delta_{r}=(2 / 3) \min \left\{\Delta_{c} ; \Delta_{v}\right\}$ and $\Delta_{d}=V_{0}$. It does not apply to electrons, for which $\Delta_{d}$ $=\Delta_{c}-V_{0}$. The effect is sufficient to cause the increase in photoconductivity.
In inhomogeneous crystalline semiconductors, the photoconductivity increases as the temperature decrease. In polymer-based structures, this may not be the case in spite of the explicit exponential factors in Eqs. (11), (13), and (15). The hopping character of polymer conductivity implies a mobility $\mu_{p}$ with an activation temperature dependence of opposite sign, so that the total character of $\sigma_{p}$ vs $T$ dependence remains indefinite.

It is worthwhile to follow the change in photoconductivity with the concentration of inclusions $N$. Equations (11) and (15) show a weak increase of $\sigma_{p}$ with $N$. This occurs because the drift barrier for holes is lowered as the interinclusion distance is decreased and the height of potential profile $V_{0}$ lowered. On the other hand, if carriers are generated in the wide-gap matrix but recombine in the narrow-gap inclusions, then the recombination rate increases with $N$. The corresponding decrease in carrier concentration compensates the above-mentioned decrease of $\Delta_{d}$ so that $\sigma_{p}$ remains independent of $N$ [see Eq. (13)].

Qualitative changes occur at $4 \pi \mathrm{Na}^{3} / 3 \simeq 0.2$ when percolation through inclusions becomes possible. At larger $N$, electrons can move from one inclusion to another across the whole sample, and the drift barrier for electrons disappears. The drift barrier for holes also disappears since the regions of maximal hole concentration lie near the matrix-inclusion interface and become connected simultaneously with inclusions. At the same time, electrons and holes remain spatially separated and the recombination barrier is still determined by the band offsets. As result, at this critical concentration of inclusions, the partial photoconductivities of both electrons and holes (especially, electrons), and, hence the total photoconductivity, must show a marked increase.

\section{QUANTUM YIELD OF A PHOTODIODE}

In this section we calculate the quantum yield $\eta$ of a polymer-based photodiode structure. We assume that the photosensitive material occupies the region $0<x<L$ with the electron-injecting contact at $x=0$ and the hole-injecting contact at $x=L$. The voltage $U$ is applied between contacts, giving rise to the internal electric field $E=U / L$.

For comparison, we consider a homogeneous polymer containing no inclusions. For a sufficiently large field, we can neglect carrier diffusion and focus on drift. We write the continuity equations for electrons and holes

$$
\begin{aligned}
& \mu_{n} E \frac{d n}{d x}=-\gamma n p+G ; \\
& -\mu_{p} E \frac{d p}{d x}=-\gamma n p+G
\end{aligned}
$$

to be solved with the boundary conditions

$$
n(L)=0 ; \quad p(0)=0
$$

describing fast recombination of carriers at the respective contacts.

Integration of the system Eqs. (16) and (17) yield

$j=e\left(\mu_{n} n+\mu_{p} p\right) E=\operatorname{const}(x)$ 
indicating continuity of the total electric current. Eliminating $p$ from Eq. (16) with the help of Eq. (19), we obtain a firstorder differential equation for $n(x)$. It is readily integrated, and the boundary conditions Eq. (18) are used to determine the integration constant and the unknown parameter $j$. This provides an expression for the quantum yield $\eta=j /(e G L)$ as an implicit function of a single parameter $\xi$ $=\sqrt{\mu_{n} \mu_{p}} E /(\sqrt{\gamma G} L)$

$$
4 \arctan \left(\frac{\eta}{\sqrt{4 \xi^{2}-\eta^{2}}}\right)=\frac{\sqrt{4 \xi^{2}-\eta^{2}}}{\xi^{2}} .
$$

It can be shown from Eq. (20) that when $\xi \ll 1, \eta \simeq 2 \xi$, whereas when $\xi \gg 1$, the quantum yield tends to unity. The parameter $\xi$ has a simple physical interpretation. It is a ratio of the characteristic recombination time to the average time for carriers to drift between contacts.

It is fruitful to compare Eq. (20) with the quantum yield of photoresistors $\eta_{\text {res }}=\left(\mu_{n}+\mu_{p}\right) E /(\sqrt{\gamma G} L)$ taken for the same $E$. Based on the asymptotic behavior of Eq. (20), we can see that these quantum yields coincide for small $E$ in symmetric structures with $\mu_{n}=\mu_{p}$, but in all other cases $\eta_{\text {res }}>\eta$. This comparison, however, is in many cases is purely formal since, in contrast with resistors in which the electric field $E$ is created by an external voltage, the electric field in photodiodes us related to the built-in field of a $p-n$ junction.

Our analysis applies not only to conducting polymers, but to semiconductors as well. However, in real crystalline semiconductors, as a rule, $\xi \gg 1$, and the problem of quantum yield does not exist (except for the case of anomalously high density of recombination centers ${ }^{15}$ ). In contradistinction, in polymers, which carrier mobilities are typically very low, $\xi$ $\ll 1$ and quantum yield is typically very low.

Now we apply the general formula of the present section to describe photovoltaic effects in composite structures. To do this, we generalize the results of Sec. II to the case of macroscopically nonuniform systems in which the concentrations of nonequilibrium carriers $Q$ and $p_{0}$ vary along the direction of built-in electric field. For this reason we no longer assume local neutrality as expressed by Eq. (3), but consider $Q$ and $p_{0}$ as independent variables which different spatial profiles.

We assume that the total thickness of the structure $L$ is much greater than the distance $l$ between inclusions. In this case we may divide our system into domains larger than $l$ but considerably smaller than $L$ and calculate their properties by a spatial averaging. Unless the concentration of inclusions $N$ is very small, the average electron concentration will be determined purely by the inclusions: $\bar{n}=Q N / e$. The hole concentration is given by $\bar{p}=4 \pi \epsilon_{e} a^{4} k T N p_{0} /(e Q)$. Using the formulas of Sec. II, we obtain the average recombination rate

$$
\begin{aligned}
\mathcal{R} & =\frac{\gamma_{i} p_{0} Q N N_{v}^{i}}{e N_{v}^{e}} \exp \left(-\frac{\Delta_{v}}{k T}\right), \quad \text { for narrow-gap inclusions; } \\
& =\frac{3 \gamma_{e} p_{0} Q N_{c}^{e}}{4 \pi e a^{3} N_{c}^{i}} \exp \left(-\frac{\Delta_{c}}{k T}\right), \quad \text { for wide-gap inclusions. }
\end{aligned}
$$

The average generation rate $\mathcal{G}$ is equal to $4 \pi a^{3} N G_{i} / 3$ for generation inside inclusions and approximately $G_{e}$ for generation outside inclusions.

In analogy with Eqs. (16) and (17), the continuity equations are given by

$$
\begin{aligned}
& \frac{E}{e} \frac{d \sigma_{n}}{d x}=\mathcal{G}-\mathcal{R} ; \\
& -\frac{E}{e} \frac{d \sigma_{p}}{d x}=\mathcal{G}-\mathcal{R}
\end{aligned}
$$

with $\sigma_{n, p}$ already obtained in Sec. III and boundary conditions $Q(L)=0 ; p_{0}(0)=0$.

After the substitution of $\mathcal{R}$ and $\sigma_{n, p}$ into Eqs. (22) and (23), we obtain a set of equations for $n_{0} \equiv 3 Q /\left(4 \pi a^{3} e\right)$ and $p_{0}$ analogous to Eqs. (16) and (17), where $\mathcal{G}$ replaces $G$ and $\gamma, \mu_{n}$, and $\mu_{p}$ are replaced by the effective values

$$
\begin{gathered}
\left(\mu_{n}\right)_{\mathrm{eff}}=\mu_{n} \frac{N_{c}^{e}}{N_{c}^{i}} \exp \left(\frac{V_{0}-\Delta_{c}}{k T}\right) ; \\
\left(\mu_{p}\right)_{\mathrm{eff}}=\mu_{p} \exp \left(-\frac{V_{0}}{k T}\right) ; \\
\gamma_{\mathrm{eff}}=\gamma_{i} \frac{4 \pi a^{3} N N_{v}^{i}}{3 N_{v}^{e}} \exp \left(-\frac{\Delta_{v}}{k T}\right),
\end{gathered}
$$

for narrow-gap inclusions;

$$
=\gamma_{e} \frac{N_{c}^{e}}{N_{c}^{i}} \exp \left(-\frac{\Delta_{c}}{k T}\right) \quad \text { for wide-gap inclusions. }
$$

If we now calculate the effective parameter $\xi_{\text {eff }}$ using the values given above, we find that for wide-gap inclusions, $\xi_{\text {eff }}$ remains unchanged compared to the unperturbed polymer matrix. For narrow-gap inclusions, $\xi_{\text {eff }}$ acquires the factor $\exp \left[-\left(\Delta_{c}-\Delta_{v}\right) /(2 k T)\right]<1$. Taking into account the monotonic $\eta$ on $\xi$ given by Eq. (20), it is evident that the narrowgap inclusions decrease the quantum yield of photodiodes, whereas the wide-gap inclusions do not affect the quantum yield. This is connected with the simultaneous increase in $\left(\mu_{p}\right)_{\text {eff }}$ and decrease in $\left(\mu_{n}\right)_{\text {eff }}$ in composite structures. The qualitatively different behavior of photoconductivity and photovoltage results from the fact that photoconductivity is determined by the carriers with the higher mobility, while for the photovoltage carriers of both types are equally important.

The situation changes dramatically at the percolation threshold corresponding to $4 \pi N a^{3} / 3 \simeq 0.2$. As mentioned in Sec. III, at this density of inclusions the drift barriers disappear while the recombination barrier remains. This means that $\left(\mu_{n}\right)_{\mathrm{eff}} \simeq \mu_{n},\left(\mu_{p}\right)_{\mathrm{eff}} \simeq \mu_{p}$ whereas $\gamma_{\mathrm{eff}}$ remains exponentially small in comparison with $\gamma$ in a uniform matrix. The ultimate result is a sharp increase in $\eta$ to a value much greater than in uniform polymer structures.

\section{DISCUSSION}

In Secs. III and IV, we considered two different photosensitive structures-photoresistors and photodiodes. Their properties were shown to be influenced by second-phase in- 


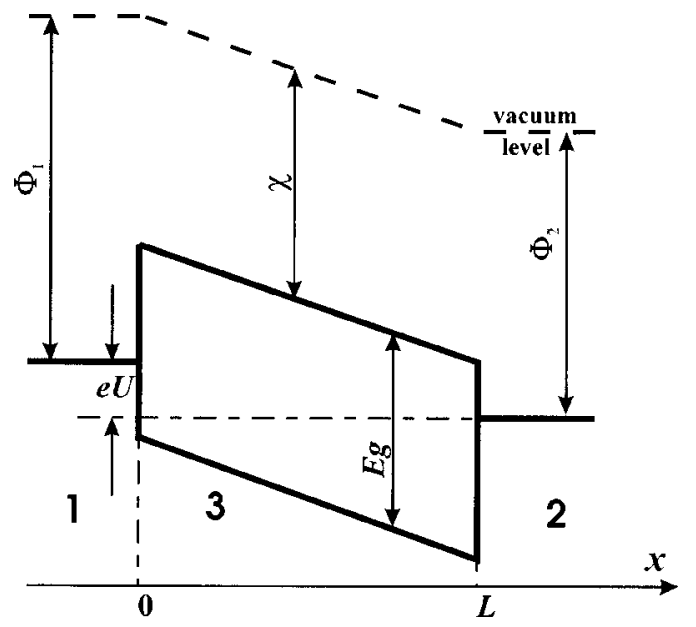

FIG. 3. The energy band diagram for a photosensitive structure. 1 and 2 are the metallic contacts with work functions $\Phi_{1}$ and $\Phi_{2} .3$ is the photosensitive layer with electron affinity $\chi$ and band gap $E_{g}$. The electric field is given by $E=\left(\Phi_{1}-\Phi_{2}+e V\right) / e$, where $V$ is the external applied voltage.

clusions in different ways. For this reason it is especially important to elucidate which of two models describe real polymer-based photosensitive structures better. These structures usually consist of a polymer layer provided with two contacts from different materials. ${ }^{5}$ The band diagram of such a system is shown schematically in Fig. 3. This system suggests a photoresistor but contains a built-in electric field resulting from the difference in contact work functions and giving rise to the short-circuit photocurrent characteristic of photodiodes. A suitable mapping between this physical system and a model thus requires clarification.

The boundary conditions, Eqs. (18), correspond to the absence of carrier injection from contacts. In this case of a pure photodiode, all carriers are optically generated in the electric field region, partially recombine, and partially reach the contacts via drift. The electron and hole concentration profiles $n(x)$ and $p(x)$ provide the continuity of electric current. In particular, $n(L)=p(0) \mu_{p} / \mu_{n}$, so that the electron current entering the right contact is equal to the hole current at the left contact. Both types of carriers play equally important role in determining the photocurrent, so that a decrease in the mobility for either of them suppresses the quantum yield.

The contacts in Fig. 3 can in fact inject both electrons and holes into a polymer layer. The relative strength of electron and hole injections for a given contact is determined by the ratio of the activational factors $\exp \left[-\left(\Phi_{1,2}-\chi\right) / k T\right]$ and $\exp \left[-\left(\chi+E_{g}-\Phi_{1,2}\right) / k T\right]$. Injection of one type of carriers decreases the influence of the other and lends the character of a photoresistor. Photocurrent exists even for $\mu_{n}=0$ since the loss of holes into the $x=0$ contact is compensated by the injection of holes at $x=L$, as in photoresistors. Photocurrent becomes equal to $e \mu_{p} E \sqrt{G / \gamma}$ as long as it remains less than the thermoionic emission from the contact, proportional to $\exp \left[-\left(\chi+E_{g}-\Phi_{2}\right) / k T\right]$. In sum, real structures with the band diagram of Fig. 3 take on the character of photoresistors, at least for a low light intensity.

Thus, from the results of Sec. III, the sensitivity of such practical devices must indeed be increased considerably by any inclusions which result in the formation of type-II heterocontacts with the polymer matrix. This conclusion is in agreement with the experimental findings summarized in Sec. I, in which a variety of different inclusions, each disparate medium forming type-II contacts with the polymer matrix, increased the photosensitivity of polymer-based structures. Moreover, in full agreement with the predictions of Sec. III, the photosensitivity exhibited a steep increase near the inclusion density corresponding to the percolation threshold.

Another important consequence of charge separation in composite structures is the slowing down of photoconductivity kinetics, also reported experimentally. ${ }^{4}$ According to Eq. (26), the coefficient of quadratic recombination $\gamma$ in the presence of inclusions acquires the additional factor $\exp \left[-\min \left\{\Delta_{c}, \Delta_{v}\right\} / k T\right]$. This will result in an exponential increase in the characteristic time of photoconductivity relaxation, which is typical for any inhomogeneous system containing recombination barriers. ${ }^{12}$

${ }^{1}$ N. C. Greenham, X. Peng, and A. P. Alivisatos, Phys. Rev. B 54, 17628 (1996).

${ }^{2}$ Y. Wang and N. Herron, J. Lumin. 70, 48 (1996).

${ }^{3}$ A. C. Arango, S. A. Carter, and P. J. Brock, Appl. Phys. Lett. 74, 1698 (1999).

${ }^{4}$ C. H. Lee, G. Yu, D. Moses, K. Pakbaz, C. Zhang, N. S. Sariciftci, A. J. Heeger, and F. Wudl, Phys. Rev. B 48, 15425 (1993).

${ }^{5}$ Organic Electroluminescent Materials and Devices, edited by S. Miyata and H. S. Nalwa (Gordon and Breach, New York, 1997).

${ }^{6}$ Handbook of Physical Quantities, edited by I. S. Grigoriev and E. Z. Meilikhov (Chemical Rubber, Boca Raton, FL, 1997).

${ }^{7}$ H. Sakaki, L. L. Chang, R. Ludeke, C.-A. Chang, G. A. Sai-Halasz, and L. Esaki, Appl. Phys. Lett. 31, 211 (1977).

${ }^{8}$ S. F. Musikhin, L. G. Bakueva, V. I. Il'in, O. V. Rabiso, and L. V. Sharonova, Superlattices Microstruct. 15, 495 (1994).

${ }^{9}$ D. R. Frankl, Electrical Properties of Semiconductor Surfaces (Pergamon, New York, 1967).

${ }^{10}$ M. C. Schlmap, X. Peng, and A. P. Alivisatos, J. Appl. Phys. 82, 5837 (1997).

${ }^{11}$ H. Matoussi, L. H. Radzilowski, B. O. Dabbousi, E. L. Thomas, M. G. Bawendi, and M. F. Rubner, J. Appl. Phys. 83, 7965 (1998).

${ }^{12}$ A. Shik, Electronic Properties of Inhomogeneous Semiconductors (Gordon and Breach, New York, 1995).

${ }^{13}$ B. I. Shklovskii and A. L. Efros, Electronic Properties of Doped Semiconductors (Springer, New York, 1986).

${ }^{14}$ B. I. Shklovskii, JETP Lett. 14, 269 (1971).

${ }^{15}$ L. V. Asryan and A. Y. Shik, Sov. Phys. Semicond. 22, 383 (1988). 